\title{
A study on a feedforward neural network to solve partial differential equations in hyperbolic-transport problems ${ }^{\star}$
}

\author{
Eduardo Abreu ${ }^{1[0000-0003-1979-3082]}$ and Joao B. Florindo ${ }^{1[0000-0002-0071-0227]}$ \\ Institute of Mathematics, Statistics and Scientific Computing - University of \\ Campinas, Rua Sérgio Buarque de Holanda, 651, Cidade Universitária "Zeferino Vaz" \\ - Distr. Barão Geraldo, CEP 13083-859, Campinas, SP, Brasil \\ http://www.ime.unicamp.br \\ \{eabreu,florindo\}@unicamp.br
}

\begin{abstract}
In this work we present an application of modern deep learning methodologies to the numerical solution of partial differential equations in transport models. More specifically, we employ a supervised deep neural network that takes into account the equation and initial conditions of the model. We apply it to the Riemann problems over the inviscid nonlinear Burger's equation, whose solutions might develop discontinuity (shock wave) and rarefaction, as well as to the classical onedimensional Buckley-Leverett two-phase problem. The Buckley-Leverett case is slightly more complex and interesting because it has a non-convex flux function with one inflection point. Our results suggest that a relatively simple deep learning model was capable of achieving promising results in such challenging tasks, providing numerical approximation of entropy solutions with very good precision and consistent to classical as well as to recently novel numerical methods in these particular scenarios.
\end{abstract}

Keywords: Neural networks - Partial differential equation - Transport models · Numerical approximation methods for PDEs · Approximation of entropy solutions.

\section{Introduction}

Deep learning techniques have been applied to a variety of problems in science during the last years, with numerous examples in image recognition [12, natural language processing [24, self driving cars 7], virtual assistants [14, healthcare [16], and many others. More recently, we have seen a growing interest on applying those techniques to the most challenging problems in mathematics and the solution of differential equations, especially partial differential equations (PDE), is a canonical example of such task [19].

Despite the success of recent learning-based approaches to solve PDEs in relatively "well-behaved" configurations, we still have points in these methodologies

\footnotetext{
* Supported by São Paulo Research Foundation (FAPESP), National Council for Scientific and Technological Development, Brazil (CNPq), and PETROBRAS - Brazil.
} 
and applications that deserve more profound discussion, both in theoretical and practical terms. One of such points is that many of these models are based on complex structures of neural networks, sometimes comprising a large number of layers, recurrences, and other "ad-hoc" mechanisms that make them difficult to be trained and interpreted. Furthermore, we have seen little discussion on more challenging problems, like those involving discontinuities and "shock" solutions numerical approximation of entropy solutions in hyperbolic-transport problems, see, e.g., 3/8|913 1723|2 10, and references cited therein.

This is the motivation for the study accomplished in this work, where we investigate a simple feed-forward architecture, based on the physics-informed model proposed in [19, to complex problems involving PDEs in transport models. More specifically, we analyze the numerical solutions of four initial-value problems: three problems on the inviscid nonlinear Burgers PDE (involving shock wave and smooth/rarefaction fan for distinct initial conditions) and on the onedimensional Buckley-Leverett equation for two-phase configuration, which is a litle rather more complex and interesting because it has a non-convex flux function with one inflection point. The neural network consists of 9 stacked layers with tanh activation and geared towards minimizing the approximation error both for the initial values and for values of the PDE functional calculated by automatic differentiation.

The achieved results are promising. We managed to obtain an average quadratic error of $0.0005,0.0018,0.0001$, and 0.0021 , respectively, for the rarefaction, shock, smooth, and Buckley-Leverett problems. Such results are pretty interesting if we consider the low complexity of the neural model and the challenge involved in these discontinuous cases. It also strongly suggests more in-depth studies on deep learning models that account for the underlying equation. They seem to be a quite promising line to be explored for challenging problems arising in physics, engineering, and many other areas.

\section{Hyperbolic problems in transport models}

Hyperbolic partial differential equations in transport models describe a wide range of wave-propagation and transport phenomena arising from scientific and industrial engineering area. This is a fundamental research that is in active progress since it involves complex multiphysics and advanced simulations due to a lack of general mathematical theory for closed-analytical solutions (see, e.g., [1315/513 82319] and references cited therein). A basic fact of nonlinear hyperbolic transport problems is the possible loss of regularity in their solutions, namely, even solutions which are initially smooth (i.e., initial datum) may become discontinuous within finite time (blow up in finite time) and after this singular time, nonlinear interaction of shocks and rarefaction waves will play an important role in the dynamics. For the sake of simplicity and without loss of generality, we consider the scalar 1D Cauchy problem

$$
\frac{\partial u}{\partial t}+\frac{\partial H(u)}{\partial x}=0, \quad x \in \mathbb{R}, \quad t>0, \quad u(x, 0)=u_{0}(x),
$$


here $H \in C^{2}(\Omega), H: \Omega \rightarrow \mathbb{R}, u_{0}(x) \in L^{\infty}(\mathbb{R})$ and $u=u(x, t): \mathbb{R} \times \mathbb{R}^{+} \longrightarrow$ $\Omega \subset \mathbb{R}$. In several applications, the flux function $H(u)$ is smooth and with a finite number of inflection points, namely $H(u)=\frac{u^{2}}{u^{2}+a(1-u)^{2}}, 0<a<1$, such as is the case of classical Buckley-Leverett equation (it has a non-convex flux function with one inflection point) in petroleum engineering (e.g., [10]). Another interesting model is the inviscid Burgers' equation where $H(u)=u^{2} / 2$, which is used to model for example gas dynamics and traffic flow, and investigate the appearance of shock waves, especially in fluid mechanics, for nonlinear wave propagation (see, e.g., [2]).

By using an argument in terms of traveling waves to capture the viscous profile at shocks, one can conclude that solutions of (1) satisfy Oleinik's entropy condition (see e.g., [17), which are limits of solutions $u^{\epsilon}(x, t) \rightarrow u(x, t)$, where $u(x, t)$ is given by (1) and $u^{\epsilon}(x, t)$ is given by the augmented parabolic equation [18]

$$
\frac{\partial u^{\epsilon}}{\partial t}+\frac{\partial H\left(u^{\epsilon}\right)}{\partial x}=\epsilon \frac{\partial^{2} u^{\epsilon}}{\partial x^{2}}, \quad x \in \mathbb{R}, \quad t>0, \quad u^{\epsilon}(x, 0)=u_{0}^{\epsilon}(x),
$$

with $\epsilon>0$ and the same initial data as in (1).

Thus, in many situations is of importance to consider and study both model problems (1) and 22), related to hyperbolic problems in transport models. In this regard, a typical flux function $H(u)$ associated to fundamental prototype models (1) and (2) depends on the application under consideration, for instance, such as modeling slow erosion phenomena in granular flow, fluid mechanics, flow in porous media (see, e.g., [2]10]3]13] and references cited therein). Moreover, it is noteworthy that in practice calibration of function $H(u)$ can be difficult to achieve due to unknown parameters and, thus, data assimilation (i.e., regression method) can be an efficient method of calibrating these flux function $H(u)$ models [4 22. We intend to design a unified approach which combines both Partial Differential Equation (PDE) modeling and fine tuning machine learning techniques aiming as a fistt step to an effective tool for advanced simulations related to hyperbolic problems in transport models.

\subsection{A benchmark numerical scheme for solving model (1)}

From the above discussion, and for comparison purposes, we will provide correct qualitative entropy approximation solutions for model problem (1) by using two numerical schemes, namely, the conservative method

$$
U_{j}^{n+1}=U_{j}^{n}-\frac{k}{h}\left[F\left(U_{j}^{n}, U_{j+1}^{n}\right)-F\left(U_{j-1}^{n}, U_{j}^{n}\right)\right],
$$

with the associated classical Lax-Friedrichs numerical flux found elsewhere,

$$
F\left(U_{j}^{n}, U_{j+1}^{n}\right)=\frac{1}{2}\left[\frac{h}{k}\left(U_{j}^{n}-U_{j+1}^{n}\right)+\left(H\left(U_{j+1}^{n}\right)+H\left(U_{j}^{n}\right)\right)\right],
$$


as well as by the the Lagrangian-Eulerian numerical flux (21])

$$
F\left(U_{j}^{n}, U_{j+1}^{n}\right)=\frac{1}{4}\left[\frac{h}{k}\left(U_{j}^{n}-U_{j+1}^{n}\right)+2\left(H\left(U_{j+1}^{n}\right)+H\left(U_{j}^{n}\right)\right)\right] .
$$

Here, both schemes (4) and (5) should follow the stability Courant-FriedrichsLewy (CFL) condition

$$
\max _{j}\left\{\left|H^{\prime}\left(U_{j}^{n}\right)\right|\right\} \frac{k}{h}<\frac{1}{2},
$$

for all time steps $n$, where $k=\Delta t^{n}$ and $h=\Delta x, H^{\prime}\left(U_{j}^{n}\right)$ is the partial derivative of $H$, namely $\frac{\partial H(u)}{\partial u}$ for all $U_{j}^{n}$ in the mesh grid.

\section{Related works}

We are interested in the study of a unified approach which combines both data-driven models (regression method by machine learning) and physics-based models (PDE modeling). Our approach is substantially distinct from the current trend of merely data-driven discovery type methods for recovery governing equations by using machine learning and artificial intelligence algorithms in a straightforward manner. We glimpse the use of novel methods, fine tuning machine learning algorithms and very fine mathematical and numerical analysis to improve comprehension of regression methods aiming to identify the potential and reliable prediction for advanced simulation for hyperbolic problems in transport models as well as the estimation of financial returns and economic benefits. In this regard, we mention the very interesting works [2011/4/22]19, which introduced physics-informed neural networks that are trained to solve supervised learning tasks while respecting conceptual foundations of physics as well as proving data assimilation for summarizing data-driven discovery of partial differential equations. Related to the hyperbolic problems in transport models (1) and (2), we mention the very recent review paper [6], which discusses machine learning for fluid mechanics, but highlighting that such approach could augment existing efforts for the study, modeling and control of fluid mechanics, keeping in mind the importance of honoring engineering principles and the governing equations, mathematical and physical foundations driven by unprecedented volumes of data from experiments and advanced simulations at multiple spatiotemporal scales. We also mention the work [21, where the issue of domain knowledge is addressed as a prerequisite essential to gain explainability to enhance scientific consistency from machine learning and foundations of physics-based given in terms of mathematical equations and physical laws. However, we have seen much less discussion on more challenging PDE modeling problems, like those involving discontinuities and shock' solutions numerical approximation of entropy solutions in hyperbolictransport problems, in which the issue of conservative numerical approximation of entropy solutions is crucial and mandatory [3|8|9|13|17|23|2|10|1]. 


\section{Proposed methodology}

The neural network employed here is based on that described in 19. It follows a classical feed-forward architecture, with 9 hidden layers, each one with a hyperbolic tangent used as activation function.

The general problem solved here has the form

$$
u_{t}+\mathcal{N}(u)=0, \quad x \in \Omega, t \in[0, T],
$$

where $\mathcal{N}(\cdot)$ is a non-linear operator and $u(x, t)$ is the desired solution. Unlike the methodology described in [19], here we do not have an explicit boundary condition and the neural network is optimized only over the initial conditions of each problem.

We focus on four problems: the inviscid nonlinear Burgers equation

$$
u_{t}+\left(\frac{u^{2}}{2}\right)_{x}=0, \quad x \in[-10,10], \quad t \in[0,8],
$$

with shock initial condition

$$
u(x, 0)=1, x<0 \text { and } u(x, 0)=0, x>0,
$$

discontinuous initial data (hereafter rarefaction fan initial condition)

$$
u(x, 0)=-1, x<0 \text { and } u(x, 0)=1, x>0,
$$

smooth initial condition

$$
u(x, 0)=0.5+\sin (x),
$$

and the two-phase Buckley-Leverett

$$
\begin{aligned}
& u_{t}+\left(\frac{u^{2}}{u^{2}+a(1-u)^{2}}\right)_{x}=0, \quad x \in[-8,8], \quad t \in[0,8], \\
& u(x, 0)=1, x<0 \text { and } u(x, 0)=0, x>0 .
\end{aligned}
$$

In this problem we take $a=1$.

For the optimization of the neural network we should define $f$ as the left hand side of each PDE, i.e.,

$$
f:=u_{t}+\mathcal{N}(u)
$$

such that

$$
\mathcal{N}(u)=\left(\frac{u^{2}}{2}\right)_{x}
$$

in the inviscid Burgers and

$$
\mathcal{N}(u)=\left(\frac{u^{2}}{u^{2}+a(1-u)^{2}}\right)_{x}
$$


in the Buckley-Leverett. Here we also have an important novelty which is the introduction of a derivative (w.r.t. $x$ ) in $\mathcal{N}(u)$, which was not present in [19].

The function $f$ is responsible for capturing the physical structure (i.e, select the qualitatively correct entropy solution) of the problem and inputting that structure as a primary element of the machine learning problem. The neural network computes the expected solution $u(x, t)$ and its output and the derivatives present in the calculus of $f$ are obtained by automatic differentiation.

Two quadratic loss functions are defined over $f, u$ and the initial condition:

$$
\begin{aligned}
& \mathcal{L}_{f}(u)=\frac{1}{N_{f}} \sum_{i=1}^{N_{f}}\left|f\left(x_{f}^{i}, t_{f}^{i},\right)\right|^{2} \\
& \mathcal{L}_{u}(u)=\frac{1}{N_{u}} \sum_{i=1}^{N_{u}}\left|u\left(x_{u}^{i}, t_{u}^{i}\right)-u^{i}\right|^{2},
\end{aligned}
$$

where $\left\{x_{f}^{i}, t_{f}^{i}\right\}_{i=1}^{N_{f}}$ correspond to collocation points over $f$, whereas $\left\{x_{u}^{i}, t_{u}^{i}, u^{i}\right\}_{i=1}^{N_{u}}$ correspond to the initial values at pre-defined points.

Finally, the solution $u(x, t)$ is approximated by minimizing the sum of both objective functions at the same time, i.e.,

$$
u(x, t) \approx \underset{u}{\arg \min }\left[\mathcal{L}_{f}(u)+\mathcal{L}_{u}(u)\right]
$$

\section{Results and Discussion}

In the following we present results for the solutions of the investigated problems obtained by the neural network model. We compare these solutions with two numerical schemes: Lagrangian-Eulerian and Lax-Friedrichs. These are very robust numerical methods with a solid mathematical basis. Here we use one scheme to validate the other. In fact, the solutions obtained by each scheme are very similar. For that reason, we opted for graphically showing curves only for the LagrangianEulerian solution. However, we exhibit the errors of the proposed methodology both in comparison with Lagrangian-Eulerian (EEL) and Lax-Friedrichs (ELF). Here such error corresponds to the average quadratic error, i.e.,

$$
\begin{aligned}
& E L F(t)=\frac{\sum_{i=1}^{N_{u}}\left(u_{N N}\left(x^{i}, t\right)-u_{L F}\left(x^{i}, t\right)\right)^{2}}{N_{u}} \\
& E E L(t)=\frac{\sum_{i=1}^{N_{u}}\left(u_{N N}\left(x^{i}, t\right)-u_{L E}\left(x^{i}, t\right)\right)^{2}}{N_{u}}
\end{aligned}
$$

where $u_{N N}, u_{L F}$, and $u_{L E}$ correspond, respectively, to the neural network, LaxFriedrichs, and Lagrangian-Eulerian solutions. In our tests, we used $N_{f}=10^{4}$ unless otherwise stated, and $N_{u}=100$. For the numerical reference schemes we adopted CFL condition 0.4 for Lax-Friedrichs and 0.2 for Lagrangian-Eulerian. We also used $\Delta x=0.01$. 
For the rarefaction case, we observed that using $N_{f}=10^{4}$ collocation points was sufficient to provide good results. In this scenario, we also verified the number of neurons, testing 40 and 60 neurons. Figure 1 shows the obtained solution compared with reference and the respective errors. Interestingly, the error decreases when time increases, which is a consequence of the solution behavior, which becomes smoother (smaller slope) for larger times, showing good accuracy and evidence that we are computing the correct solution in our numerical simulation.
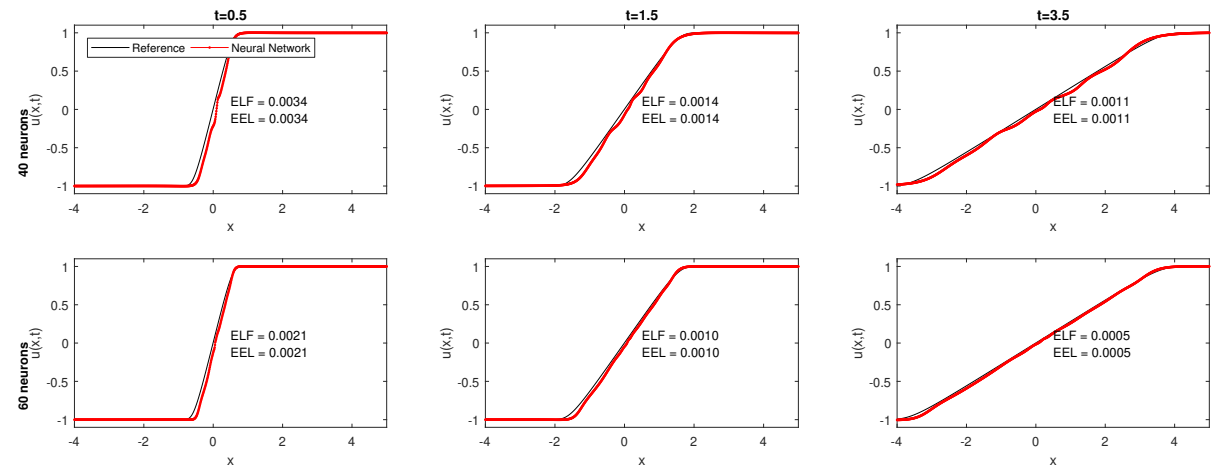

Fig. 1. Burgers: Rarefaction.

Figure 2 illustrates the performance of the neural network model for the inviscid Burgers equation with shock initial condition. Here we had to add a small viscous term $\left(0.01 u_{x x}\right)$ for better stabilization, but in view on the modeling problems (1) and (2). Here, such underlying viscous mechanism did not bring significant reduction in error, but the general structure of the obtained solution is better, attenuating spurious fluctuations around the discontinuities. It is crucial to mention at this point that numerical approximation of entropy solutions (with respect to the neural network) to hyperbolic-transport problems also require the notion of entropy-satisfying weak solution. It was also interesting to see that the addition of more neurons did not reduce the error for this initial condition. This is a typical example of overfitting caused by over-parameterization. An explanation for that is the relative simplicity of the initial condition, assuming only two possible values.

Figure 3 depicts the solutions for the smooth initial condition in the inviscid Burgers equation. Here, unlike the previous case, increasing the number of neurons actually reduced the error. And this was expected considering that now both initial condition and solution are more complex. Nevertheless, we identified that tuning only number of neurons was not enough to achieve satisfactory solution in this situation. Therefore we also tuned the parameter $N_{f}$. In particular, we discovered that combining the same small viscous term used for the shock 
Abreu and Florindo
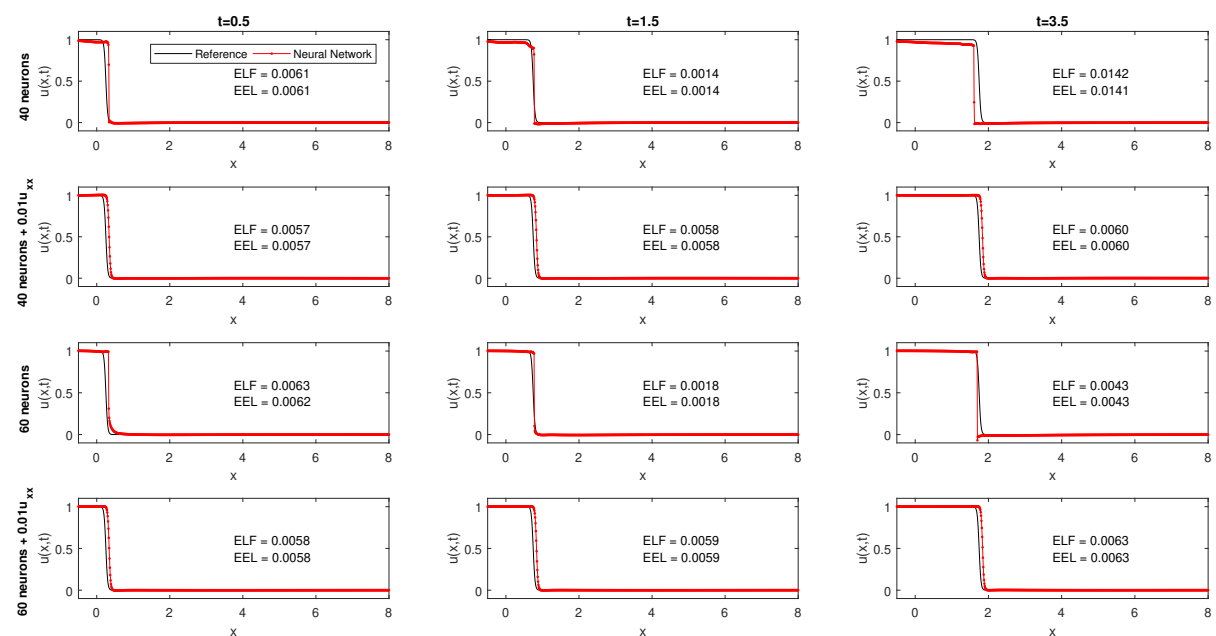

Fig. 2. Burgers: Shock.

case with $N_{f}=10^{6}$ provided excellent results, with quite promising precision in comparison with our reference solutions.
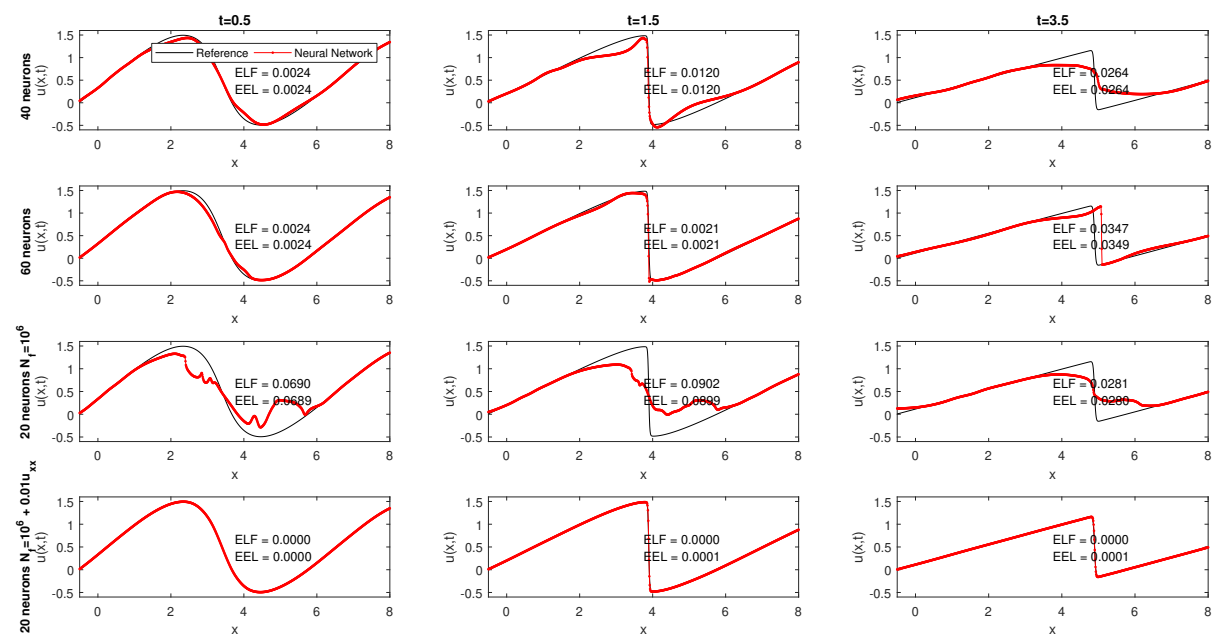

Fig. 3. Burgers: Smooth.

Another case characterized by solutions with more complex behavior is BuckleyLeverett with shock initial condition (Figure 4). And, similar to what happened in the smooth case, again, the combination of $N_{f}=10^{6}$ with the small viscous term was more effective than any increase in the number of neurons. While the 
introduction of the small viscous term attenuated fluctuations in the solution when using 40 neurons, at the same time when using $N_{f}=10^{4}$, we observe that increasing the number of neurons causes an increase in the delay between the solution provided by the network and the reference.
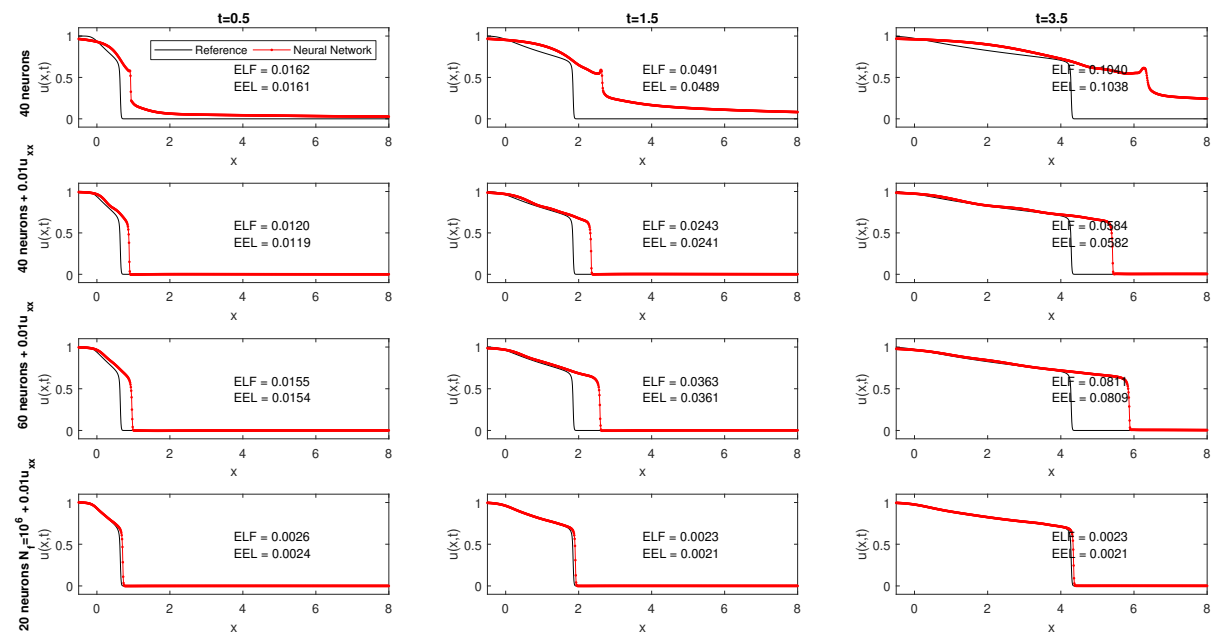

Fig. 4. Buckley-Leverett: Rarefaction + Shock.

Generally speaking, the neural networks here studied were capable of achieving promising results in challenging situations, involving different types of discontinuities and nonlinearities. Moreover, our results also bring important theoretical implications. In particular, the neural networks obtained results pretty close to those provided by entropic numerical schemes like Lagrangian-Eulerian and Lax-Friedrichs. Going beyond the analysis in terms of raw precision, these results give us evidences that our neural network model possess some type of entropic property, which from the viewpoint of a numerical method is a fundamental and desirable characteristic.

\section{Conclusions}

This work presented an application of a feed-forward neural network to solve challenging hyperbolic problems in transport models. More specifically, we solve the inviscid Burgers equation with shock, smooth and rarefaction initial conditions, as well as the Buckley-Leverett equation with classical Riemann datum, which lead to the well-known solution that comprises (from left to right) a rarefaction and a (discontinuous) shock wave. Our network was tuned according to each problem and interesting findings were observed. At first, our neural network model was capable of providing solutions pretty similar to those obtained 
by two numerical schemes here used as references: Lagrangian-Eulerian and LaxFriedrichs. Besides, the general structure of the obtained solutions also behaved as expected, which considering the intrinsic challenge of these problems is a remarkable achievement. In fact, the investigated neural networks showed evidences of an entropic property, which is an important attribute of a numerical scheme, especially in problems like those investigated here.

In summary, the obtained results share both practical and theoretical implications. In practical terms, the results confirm the potential of a relatively simple deep learning model in the solution of an intricate numerical problem. In theoretical terms, this also opens an avenue for formal as well as rigorous studies on these networks as mathematically valid and effective numerical methods.

\section{Acknowledgements}

J. B. Florindo gratefully acknowledges the financial support of São Paulo Research Foundation (FAPESP) (Grant \#2016/16060-0) and from National Council for Scientific and Technological Development, Brazil (CNPq) (Grants \#301480 /2016-8 and \#423292/2018-8). E. Abreu gratefully acknowledges the financial support of São Paulo Research Foundation (FAPESP) (Grant \#2019/20991-8), from National Council for Scientific and Technological Development - Brazil (CNPq) (Grant \#2 306385/2019-8) and PETROBRAS - Brazil (Grant \#2015/ 00398-0). E. Abreu and J. B. Florindo also gratefully acknowledge the financial support of Red Iberoamericana de Investigadores en Matemáticas Aplicadas a Datos (MathData).

\section{References}

1. Abreu, E., Matos, V., Pérez, J., Rodríguez-Bermúdez, P.: A class of lagrangianeulerian shock-capturing schemes for first-order hyperbolic problems with forcing terms. Journal of Scientific Computing (to appear) (2021)

2. Abreu, E., Pérez, J.: A fast, robust, and simple lagrangian-eulerian solver for balance laws and applications. Computers \& Mathematics with Applications 77(9), 2310 - 2336 (2019)

3. Alibaud, N., Andreianov, B., Ouédraogo, A.: Nonlocal dissipation measure and $1^{1}$ kinetic theory for fractional conservation laws. Communications in Partial Differential Equations 45(9), 1213-1251 (2020)

4. Berg, J., Nyström, K.: Data-driven discovery of pdes in complex datasets. Journal of Computational Physics 384, 239 - 252 (2019)

5. Bressan, A., Chiri, M.T., Shen, W.: A posteriori error estimates for numerical solutions to hyperbolic conservation laws (2020), Available at https://arxiv. org/abs/2010.00428

6. Brunton, S.L., Noack, B.R., Koumoutsakos, P.: Machine learning for fluid mechanics. Annual Review of Fluid Mechanics 52(1), 477-508 (2020)

7. Chen, C., Seff, A., Kornhauser, A., Xiao, J.: DeepDriving: Learning Affordance for Direct Perception in Autonomous Driving. In: 2015 IEEE International Conference on Computer Vision (ICCV). pp. 2722-2730. IEEE International Conference on Computer Vision (2015) 
8. Chen, G.Q.G., Glimm, J.: Kolmogorov-type theory of compressible turbulence and inviscid limit of the navier-stokes equations in r3. Physica D: Nonlinear Phenomena 400, 132138 (2019)

9. Dafermos, C.M.: Hyperbolic conservation laws in continuous physics. Springer (2016)

10. Galvis, J., Abreu, E., Díaz, C., Pérez, J.: On the conservation properties in multiple scale coupling and simulation for darcy flow with hyperbolic-transport in complex flows. Multiscale Modeling and Simulation 18(4), 1375-1408 (2020)

11. Grossi, M.D., Kubat, M., Özgökmen, T.M.: Predicting particle trajectories in oceanic flows using artificial neural networks. Ocean Modelling 156, 101707 (2020)

12. He, K., Zhang, X., Ren, S., Sun, J.: Deep Residual Learning for Image Recognition. In: 2016 IEEE Conference on Computer Vision and Pattern Recognition (CVPR). pp. 770-778. IEEE Conference on Computer Vision and Pattern Recognition (2016)

13. Hoel, H., Karlsen, K.H., Risebro, N.H., Storrosten, E.B.: Numerical methods for conservation laws with rough flux. Stochastics and Partial Differential EquationsAnalysis and Computations 8(1), 186-261 (2020)

14. Kepuska, V., Bohouta, G.: Next-Generation of Virtual Personal Assistants (Microsoft Cortana, Apple Siri, Amazon Alexa and Google Home). In: 2018 IEEE 8TH Annual Computing and Communication Workshop and Conference (CCWC). pp. 99-103 (2018)

15. Lellis, C.D., Kwon, H.: On non-uniqueness of hölder continuous globally dissipative euler flows (2020), Available at https://arxiv.org/abs/2006.06482

16. Litjens, G., Kooi, T., Bejnordi, B.E., Setio, A.A.A., Ciompi, F., Ghafoorian, M., van der Laak, J.A.W.M., van Ginneken, B., Sanchez, C.I.: A survey on deep learning in medical image analysis. Medical Image Analysis 42, 60-88 (2017)

17. Oleinik, O.A.: Discontinuous solutions of nonlinear differential equations. Uspekhi Matematicheskikh Nauk, Vol. 12, 1957, pp. 3-73. (English translation Transactions of the American Mathematical Society 26(2), 95-172 (1963)

18. QUINN, B.: Solutions with shocks: an example of an $\mathrm{L}_{1}$-contraction semigroup. Communications on Pure and Applied Mathematics 24(2), 125-132 (1971)

19. Raissi, M., Perdikaris, P., Karniadakis, G.: Physics-informed neural networks: A deep learning framework for solving forward and inverse problems involving nonlinear partial differential equations. Journal of Computational Physics 378, 686 707 (2019)

20. Regazzoni, F., Dedè, L., Quarteroni, A.: Machine learning of multiscale active force generation models for the efficient simulation of cardiac electromechanics. Computer Methods in Applied Mechanics and Engineering 370, 113268 (2020)

21. Roscher, R., Bohn, B., Duarte, M.F., Garcke, J.: Explainable machine learning for scientific insights and discoveries. IEEE Access 8, 42200-42216 (2020). https://doi.org/10.1109/ACCESS.2020.2976199

22. Rudy, S.H., Brunton, S.L., Proctor, J.L., Kutz, J.N.: Data-driven discovery of partial differential equations. Science Advances 3(4) (2017)

23. Serre, D., Silvestre, L.: Multi-dimensional burgers equation with unbounded initial data: Well-posedness and dispersive estimates. Archive for Rational Mechanics and Analysis 234, 1391-1411 (2019)

24. Young, T., Hazarika, D., Poria, S., Cambria, E.: Recent Trends in Deep Learning Based Natural Language Processing. IEEE Computational Intelligence Magazine 13(3), 55-75 (2018) 